\title{
Direct evidence of reduced dynamic scattering in the lower polariton of a semiconductor microcavity
}

Borri, Paola; Jensen, Jacob Riis; Langbein, Wolfgang; Hvam, Jørn Märcher

Published in:

Physical Review B Condensed Matter

Link to article, DOI:

10.1103/PhysRevB.61.R13377

Publication date:

2000

Document Version

Publisher's PDF, also known as Version of record

Link back to DTU Orbit

Citation (APA):

Borri, P., Jensen, J. R., Langbein, W., \& Hvam, J. M. (2000). Direct evidence of reduced dynamic scattering in the lower polariton of a semiconductor microcavity. Physical Review B Condensed Matter, 61(20), R13377R13380. https://doi.org/10.1103/PhysRevB.61.R13377

\section{General rights}

Copyright and moral rights for the publications made accessible in the public portal are retained by the authors and/or other copyright owners and it is a condition of accessing publications that users recognise and abide by the legal requirements associated with these rights.

- Users may download and print one copy of any publication from the public portal for the purpose of private study or research.

- You may not further distribute the material or use it for any profit-making activity or commercial gain

- You may freely distribute the URL identifying the publication in the public portal 


\title{
Direct evidence of reduced dynamic scattering in the lower polariton of a semiconductor microcavity
}

\author{
P. Borri, ${ }^{*}$ J. R. Jensen, W. Langbein, ${ }^{*}$ and J. M. Hvam \\ Research Center COM, The Technical University of Denmark, Building 349, DK-2800 Kgs. Lyngby, Denmark
}

(Received 21 December 1999; revised manuscript received 7 February 2000)

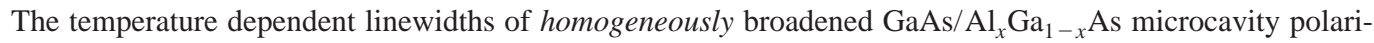
tons are investigated. The linewidths of the lower, middle, and upper polariton resonances are measured directly from reflection spectra at normal incidence $\left(k_{\|}=0\right)$. The linewidth of the lower polariton is found to be smaller than the linewidths of the middle and upper polaritons at all investigated temperatures ranging from 11 to $100 \mathrm{~K}$. The results clearly show the reduction of dynamic scattering processes in the lower polariton compared to the middle and upper polaritons, in agreement with theoretical predictions in literature. A nontrivial temperature dependence of the linewidth is found and its physical origin is discussed.
\end{abstract}

The optical linewidth of polariton resonances in semiconductor quantum-well microcavities has been the subject of intense theoretical and experimental work in recent years. Particular attention has been devoted to the role of the static structural disorder in the optical response of semiconductor microcavities in the strong coupling regime. This regime occurs when the damping mechanisms are weak compared to the exciton-photon coupling, resulting in a normal mode energy splitting when the cavity mode is close to the exciton resonance. It has been found experimentally ${ }^{1,2}$ that at resonance the inhomogeneous linewidth of the lower polariton is narrower than that of the upper polariton. This has been explained by a spatial averaging over the disorder potential in the lower polariton ${ }^{1,3,4}$ (motional narrowing) or by a linear dispersion theory together with an asymmetric lineshape of the bare quantum well (QW) excitonic absorption. ${ }^{2}$ The role of phonon scattering and polariton-polariton scattering in the homogeneous polariton linewidth has also been recently discussed. Polariton-polariton scattering is predicted to be inhibited in the lower polariton resonance at $k_{\|}=0$ as long as the scattering involves only the small region of $k$ space, where the polariton effective mass is very light due to the photon coupling, resulting in a very small density of states. 5 This leads to a threshold density for the polariton-polariton scattering in the lower polariton branch. ${ }^{5}$ Experimental results confirming this prediction have been reported. ${ }^{6,7}$ However, due to the inhomogeneous broadening of the investigated samples, the homogeneous linewidth of the polaritons had to be inferred from nontrivial nonlinear experiments. Polariton-acoustic phonon scattering is also predicted to be strongly quenched in the lower polariton branch, as long as the temperature is low enough to limit the interaction to the zone where the density of states is very small. ${ }^{8}$ Indications of this behavior have been experimentally shown ${ }^{9,10}$ but again using indirect techniques, based on interferometric timecorrelated secondary emission on inhomogeneously broadened samples. Moreover, no direct comparison between the lower and upper polariton homogeneous broadenings was presented.

In this work we have investigated the temperaturedependent linewidth of homogeneously broadened microcavity polaritons in the strong coupling regime using white-light reflection spectra at normal incidence $\left(k_{\|}=0\right)$. The sample consists of an MBE-grown $25 \mathrm{~nm} \mathrm{GaAs} / \mathrm{Al}_{0.3} \mathrm{Ga}_{0.7} \mathrm{As}$ single quantum well placed in the center of a $\lambda$ cavity. An AlAs $/ \mathrm{Al}_{0.15} \mathrm{Ga}_{0.85} \mathrm{As}$ Bragg reflector of 25 (16) periods was grown at the bottom (top) of the cavity. The $\mathrm{Al}_{0.3} \mathrm{Ga}_{0.7} \mathrm{As}$ barriers are $5 \mathrm{~nm}$ thick and the rest of the spacer layer consists of $\mathrm{Al}_{0.05} \mathrm{Ga}_{0.95} \mathrm{As}$. The spacer layer is wedged, in order to tune the cavity mode along the position on the sample. Details on the growth conditions and sample design can be found in Ref. 11. The white-light source was focused on the sample with a spot diameter of $75 \mu \mathrm{m}$, resulting in a variation of the cavity resonance over the spot size of only 100 $\mu \mathrm{eV}$, due to the wedged geometry. This variation is more than a factor of 2 smaller than the cavity linewidth, as shown in the following. Smaller spot diameters resulted in an increased cavity linewidth due to larger incident $k$ vectors. The sample was held in an exchange gas cryostat, at temperatures from $T=11$ to $100 \mathrm{~K}$. For $T<40 \mathrm{~K}$, the spectra were detected using a high resolution spectrometer and a CCD camera, with a spectral resolution of $15 \mu \mathrm{eV}$ half width at half maximum (HWHM). Above $40 \mathrm{~K}$, a normal resolution spectrometer was used resulting in $50 \mu \mathrm{eV}$ HWHM resolution. All the linewidths will be given in the paper as HWHM.

The use of a wide GaAs quantum well leads to a homogeneously broadened QW exciton absorption resonance, as experimentally shown in Ref. 12, due to the small effect of interface roughness on the confined excitons. An absorption linewidth of $60 \mu \mathrm{eV}$ at $5 \mathrm{~K}$ is found at the $1 s$ heavy-hole exciton, dominated by radiative decay. ${ }^{12}$ The exciton-photon coupling in the microcavity structure leads to three polariton resonances, arising from the mixing of the heavy-hole $(\mathrm{HH})$ exciton, light-hole (LH) exciton, and cavity modes. Measurements at $11 \mathrm{~K}$ of the polariton resonances at different detuning between the cavity mode and the $\mathrm{HH}$ exciton can be fitted by a three coupled-oscillator model from which we have inferred a Rabi splitting of $3.6 \mathrm{meV}$ for the $\mathrm{HH}$ and $2.2 \mathrm{meV}$ for the $\mathrm{LH}$ excitons. ${ }^{11}$ The temperature dependence of the reflectivity spectra for a tuning of the cavity mode approximately in resonance with the $\mathrm{HH}$ exciton is shown in Fig. 1. The spectra have been corrected for the temperature induced band-gap shifts by aligning the reflectivity minima of the lower polariton (LP), for better clarity. It can be clearly seen that the middle (MP) and upper polariton (UP) 


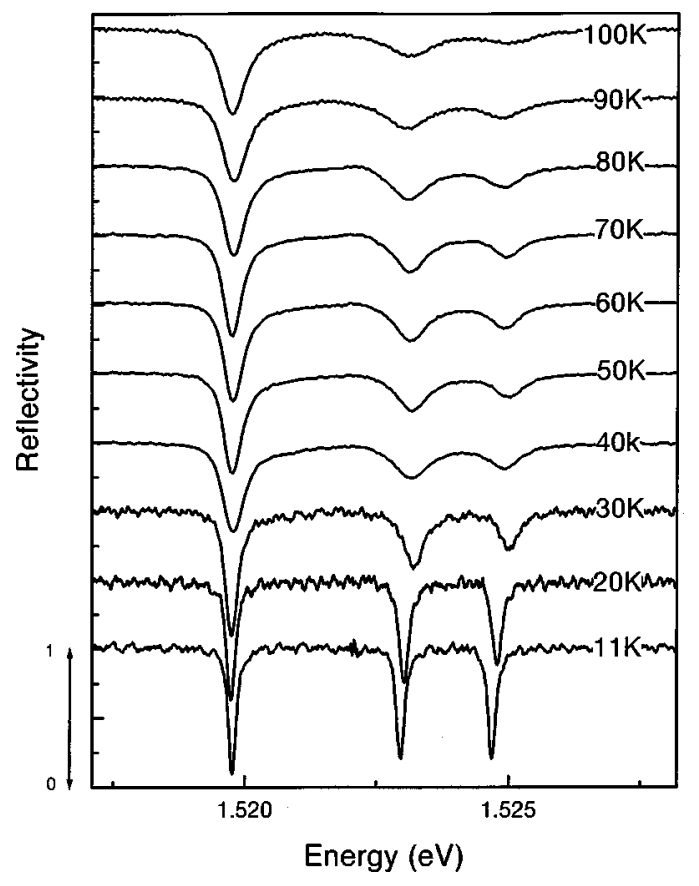

FIG. 1. Reflectivity spectra near zero detuning at different temperatures as indicated. The energy positions are shifted to overlap at the LP resonance for better clarity.

linewidths are strongly broadened by increasing the temperature, while the LP is narrower than MP and UP for $T>11 \mathrm{~K}$. The reflectivity spectrum at $11 \mathrm{~K}$ is fitted with a Lorentzian lineshape at each resonance, as shown in Fig. 2. A linewidth of $95 \mu \mathrm{eV}$ at each of the resonances is found. At higher temperatures, the linewidth broadening leads to a partial merging of the polariton line shapes, especially for the MP and UP. In this case we have fitted the reflectivity spectrum using a triple-correlated Lorentzian function, according to the following expression:

$$
R=1-\left|\sum_{n=1}^{3} \frac{A_{n}}{\left(E-E_{n}\right)+i \gamma_{n}} e^{i \delta_{n}}\right|^{2},
$$

where $E_{n}(n=1,2,3)$ are the three polariton energy resonances and $\gamma_{n}$ are the corresponding linewidths. $A_{n}$ are am-

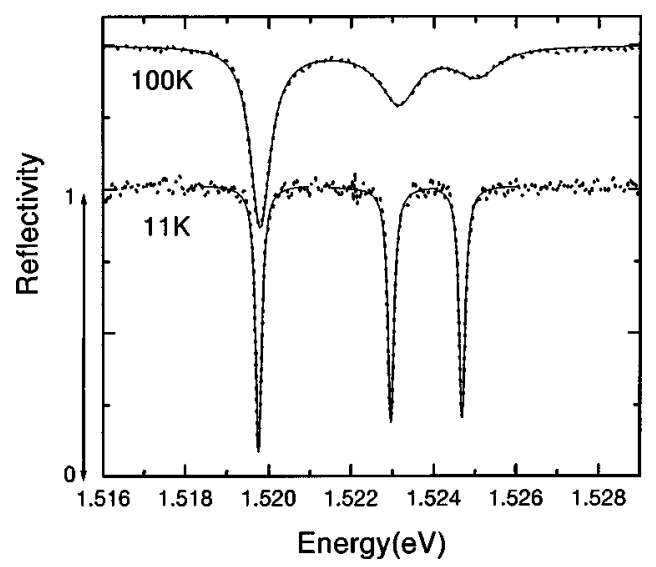

FIG. 2. Reflectivity spectra (dotted line) and fits (solid lines) at 11 and $100 \mathrm{~K}$. The fit at $11 \mathrm{~K}$ is a Lorentzian function at each resonance. The fit at $100 \mathrm{~K}$ is obtained using a triple-correlated Lorentzian function. plitude fit parameters, and $\delta_{n}$ are the relative phases.

The validity of this expression to fit the data has been checked by simulating the reflectivity spectrum in the microcavity with transfer matrix calculations. A perfect agreement between the numerical simulations and the formula used was found. The physical meaning of Eq. (1) is that the total electric field associated with the structure has to be calculated, by summing over the amplitude transmission coefficients of each resonance, to give rise to the reflectivity spectrum. ${ }^{13}$ The use of this formula to fit the spectrum at $100 \mathrm{~K}$ is shown in Fig. 2. An excellent agreement between the data and the fit is found.

Savona and Piermarocchi ${ }^{8}$ pointed out that a strong reduction in the temperature-dependent broadening of the LP linewidth compared to the UP is expected when calculating the scattering rate by acoustic-phonon absorption of the lower polariton at $k_{\|}=0$. In their work, the difference between the detuning dependence of the homogeneous linewidth resulting from the calculation and from linewidth averaging is also pointed out. The linewidth averaging approach is obtained by solving the complex eigenvalue problem of the excitonphoton coupled system ${ }^{8}$ at $k_{\|}=0$. This approach is questionable when the broadening mechanisms are related to scattering between different $k_{\|}$, and thus influenced by the different dispersion in the microcavity compared to the bare QW. ${ }^{8}$ The linewidth averaging predicts that LP and UP linewidths are equal at resonance (zero detuning) for two coupled oscillators. The calculation of Savona and Piermarocchi shows that the LP linewidth is smaller than the UP one at zero detuning. Consequently, the crossing point between the LP and UP linewidths is shifted towards negative detuning, i.e., when the cavity resonance is below the exciton resonance, assuming $\gamma_{\mathrm{HH}}<\gamma_{\mathrm{c}}$ where $\gamma_{\mathrm{HH}}$ and $\gamma_{\mathrm{c}}$ are the bare $\mathrm{HH}$ and cavity linewidths, respectively.

In Fig. 3 we show the measured detuning dependence of the linewidths for the three polariton resonances at three different temperatures, as indicated, and we compare it with the linewidth averaging in a three-coupled oscillators system. The detuning is defined as $\delta=E_{\mathrm{c}}-E_{\mathrm{HH}}$ with $E_{\mathrm{c}}$ and $E_{\mathrm{HH}}$ being the cavity and bare $\mathrm{HH}$ exciton energies. At $11 \mathrm{~K}$, a cavity linewidth of $130 \mu \mathrm{eV}$ and a bare exciton linewidth of $75 \mu \mathrm{eV}$ is deduced from the large negative detuning data. The corresponding detuning dependence expected by linewidth averaging is shown in the lower part of the figure. Due to the presence of three resonances, the calculated crossing point is not at $\delta=0$, and does not correspond to 0.5 photonic and excitonic content, as in the two-coupled oscillators model. ${ }^{8}$ Instead, the crossing point between LP and UP occurs at $\delta=1.2 \mathrm{meV}$ where the photonic content is 0.37 and the total $(\mathrm{HH}+\mathrm{LH})$ exciton content is 0.63 both in the LP and UP eigenstate. ${ }^{11}$ From our measurements at $11 \mathrm{~K}$, the crossing between the linewidths is obtained at $\delta \sim 0$. For large positive detunings, the UP linewidth, which is cavitylike, is increased by the absorption from the QW continuum. At $20 \mathrm{~K}$ a larger bare-exciton linewidth of about $90 \mu \mathrm{eV}$ is measured at negative detuning, as expected from enhanced scattering mechanisms at higher $T$ on the bare exciton. The crossing point between the LP and UP occurs at a slight negative detuning. At $70 \mathrm{~K}$ the bare exciton linewidth is above the cavity linewidth, and no crossing between the LP and UP occurs at any detuning. Therefore, the simple line- 


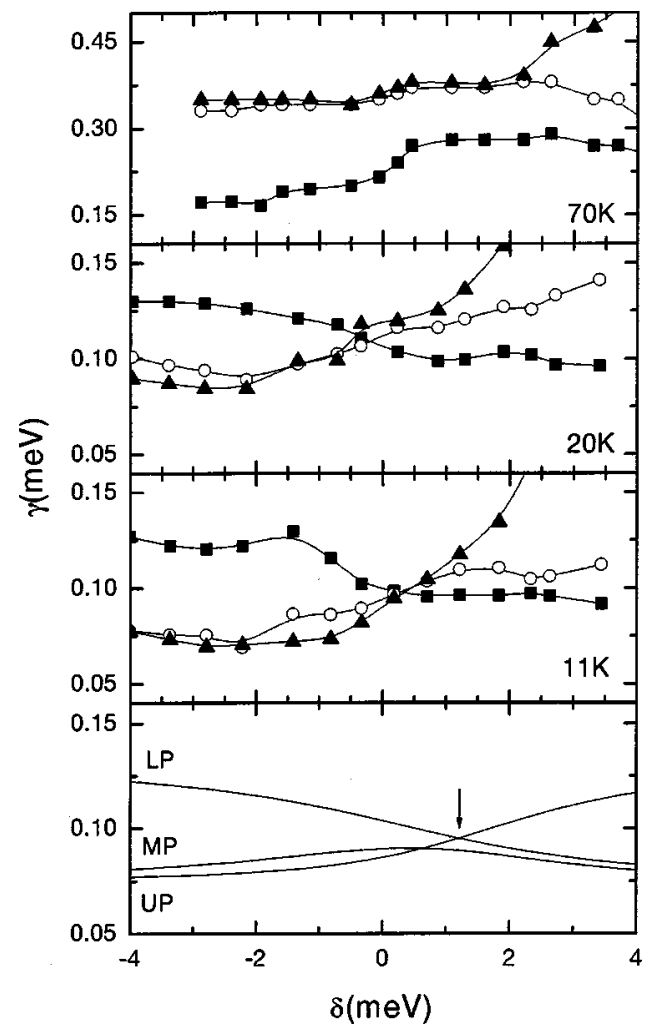

FIG. 3. LP (squares), MP (circles), and UP (triangles) linewidth versus detuning at three different temperatures as indicated. In the lower part, the detuning dependence as expected by linewidth averaging at $11 \mathrm{~K}$ is shown.

width averaging is not verified by our experimental findings at any temperature, in agreement with the discussion in Ref. 8.

The physical quantities of interest in the reduced acousticphonon absorption of the LP branch at $k_{\|}=0$ are the energy separation $\Delta E$, between the LP resonance at $k_{\|}=0$ and the $\mathrm{HH}$ bare exciton, and the cutoff $\left(q_{\mathrm{c}}\right)$ in the wave vector exchanged between the acoustic phonon and the exciton in the QW. ${ }^{8,5,10}$ The $k$ dispersion of the LP branch approaches the $\mathrm{HH}$ bare exciton for $k_{\|} \gg 0$, where a flat dispersion is recovered due to the large exciton mass. Therefore, $\Delta E$ is the energy barrier to overcome in order that the excitonlike part of the LP dispersion becomes available as final states for scattering via phonon absorption. As long as $k_{\mathrm{B}} T<\Delta E$, only a small scattering phase space is available and the broadening in the LP is negligible. ${ }^{8}$ When $k_{\mathrm{B}} T>\Delta E$, the scattering rate depends on the comparison between the acoustic phonon energy associated with $q_{\mathrm{c}}$ (i.e., $\hbar v q_{\mathrm{c}}$ with the sound velocity $v)$ and $\Delta E$. When $\hbar v q_{\mathrm{c}}<\Delta E$, the phonon scattering of the lower polariton branch is expected to be several orders of magnitude smaller than for the bare exciton. ${ }^{10,5} \mathrm{We}$ estimate $\hbar v q_{\mathrm{c}}=1.24 \mathrm{meV}$, with $v=5000 \mathrm{~ms}^{-1}$ and $^{10} q_{\mathrm{c}}=3 \pi / L$, where $L$ is the width of the quantum well. At $\delta=0$ we have $\Delta E=2 \mathrm{meV}$ and at $\delta=1.2 \mathrm{meV}, \Delta E=1.43 \mathrm{meV}$. We therefore expect in our structure a strong reduction in the acoustic-phonon absorption rate of the LP, corresponding to a nearly constant LP linewidth versus temperature until the LO-phonon absorption becomes active. The MP and UP linewidths, instead, should almost follow the temperature dependence of the bare QW exciton. ${ }^{8}$

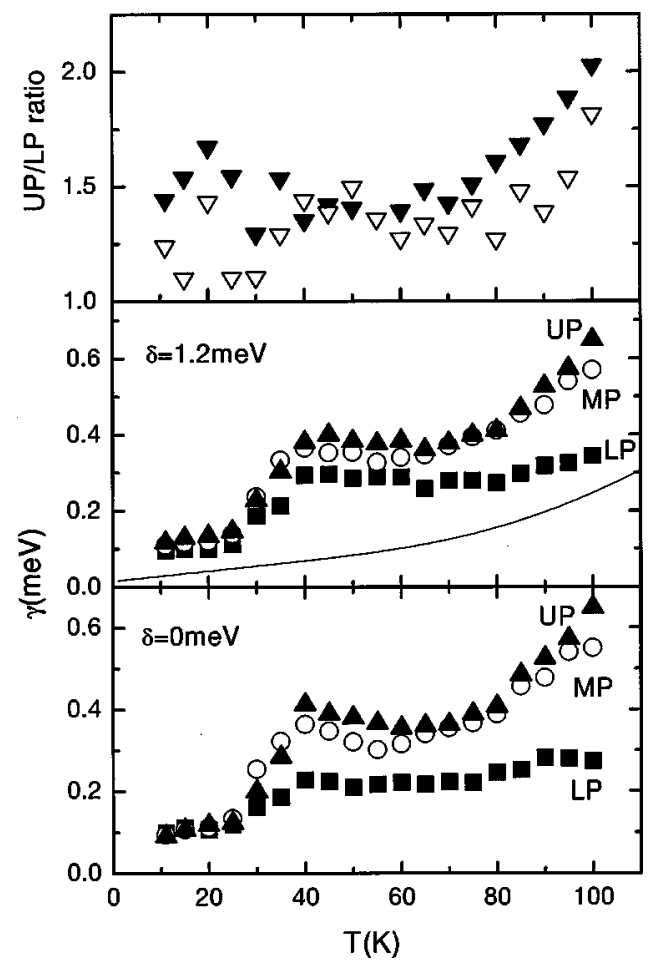

FIG. 4. Temperature dependence of the polariton linewidths for zero and $1.2 \mathrm{meV}$ detuning. The solid line is the expected linewidth dependence due to phonon absorption in the bare exciton, weighted by the excitonic content at $1.2 \mathrm{meV}$ detuning. In the top part, the ratio between the UP and LP linewidth, after subtraction of the cavity contribution, is shown at zero (open symbols) and $1.2 \mathrm{meV}$ detuning (closed symbols).

In Fig. 4 the LP, MP, and UP linewidths versus $T$ are shown at $\delta=0$ and $\delta=1.2 \mathrm{meV}$. The temperature dependence of the linewidth of the bare exciton, as expected from acoustic and optical phonon scattering is also shown (solid line), weighted by the excitonic content at $\delta=1.2 \mathrm{meV}$. An acoustic phonon coefficient of $2.1 \mu \mathrm{eV} / \mathrm{K}$, and an optical phonon coefficient of $10 \mathrm{meV}$ are used, in agreement with the measured dependence in the bare $\mathrm{QW},{ }^{12}$ and a small inhomogeneous broadening of $25 \mu \mathrm{eV}$, which is realistic for the investigated quantum well, is also included. The measured temperature dependence in the MP and UP is surprisingly quite different from the one expected by phonon scattering (even at large negative detuning where the MP and UP tend to the bare $\mathrm{HH}$ and $\mathrm{LH}$ exciton). In particular, the linewidth of MP and UP is always higher than expected, indicating that additional scattering mechanisms are taking place. We believe that an excess of free carriers in the microcavity structure, possibly due to unintentional doping of the Bragg reflectors, is responsible for a polariton scattering additional to the phonon interaction. The nonlinear increase of the linewidth up to $40 \mathrm{~K}$ could be due to an increased kinetic energy of the free carriers with temperature. Above $40 \mathrm{~K}$, the linewidth is slightly reduced up to $65 \mathrm{~K}$. This could be explained by phonon assisted escape of the excess of carriers from the $\mathrm{QW}$, via tunnelling through the shallow barriers, and consequent reduction of the free carrier concentration in the well. Finally, above $65 \mathrm{~K}$ an increased linewidth versus $T$, with a similar trend as the one due to LO-phonon absorption in the bare exciton, is found. 
We would like to point out that the investigated sample is one of the highest qualities shown up to now, with a ratio of 19 between the HH Rabi splitting and the linewidth at zero detuning. We believe that the presence of free carriers is a common problem in microcavities, often simply masked by a dominant inhomogeneous broadening. Note that Cassabois et al. ${ }^{10}$ use an acoustic phonon coefficient of $11 \mu \mathrm{eV} / \mathrm{K}$ to explain their results, much too high compared to what is reported in good-quality $\operatorname{In}_{x} \mathrm{Ga}_{1-x}$ As quantum wells, ${ }^{14}$ and possibly overestimated due to free carrier scattering. Anomalous photoinduced quenching of the polariton linewidth has been reported in $\operatorname{In}_{x} \mathrm{Ga}_{1-x}$ As quantum well microcavities, ${ }^{15}$ which could be also explained by a reduction of the free carrier density via recombination with the photoinduced carriers.

The measured temperature dependence of the polariton linewidths yields higher values than expected from pure phonon scattering, due to the additional free carrier scattering. However, we do observe a narrower linewidth in the LP compared to the MP and UP at all the investigated temperatures. It is actually reasonable that also free carrier scattering is quenched in the low polariton branch due to the general argument of a small density of states at small $k_{\|}$in the LP dispersion, which also explains a reduced polariton-polariton scattering. 5,16

In the top part of Fig. 4, the ratio between the UP and LP linewidth, after subtracting the cavity linewidth weighted by the photonic content, is shown versus temperature for $\delta=0$ and $1.2 \mathrm{meV}$. At $\delta=0$, the LP and UP do not have the same excitonic content, and the ratio has been multiplied with the ratio of the LP and UP excitonic content. According to the average linewidth model, this ratio should be 1 at all temperatures, as long as the correction to the Rabi splitting due to the broadening is negligible, ${ }^{17}$ which is applicable in our case up to $100 \mathrm{~K}$ as experimentally verified. We clearly observe that the ratio is always above 1 and tends to 2 at $100 \mathrm{~K}$. A ratio of 2 is obtained when the UP is broadened by acoustic and optical phonons of equal scattering rates, as is the case at $\sim 100 \mathrm{~K}$, while the LP is only broadened by the LO-phonon scattering. This indicates that a phononscattering picture tends to be recovered at high temperatures when the LO-phonon scattering dominates over the free carrier scattering. ${ }^{18}$

In conclusion, we have experimentally demonstrated that the LP homogeneous linewidth is narrower than the MP and UP linewidths in the strong-coupling regime and at all temperatures from 11 to $100 \mathrm{~K}$. We presented, to the best of our knowledge, the first direct evidence of reduced scattering processes in the homogeneous broadening of the LP compared to the UP. The measured temperature dependence of the linewidth shows a surprising behavior, that cannot be simply attributed to phonon scattering, and is assigned to a temperature dependent additional scattering from excess free carriers in the investigated structure.

The authors thank K. Leosson for the use of the high resolution spectrometer.
*Present address: Lehrstuhl für Experimentelle Physik EIIb, Universität Dortmund, Otto-Hahn Str.4, 44227 Dortmund, Germany.

${ }^{1}$ D. M. Whittaker, P. Kinsler, T. A. Fisher, M. S. Skolnick, A. Armitage, A. M. Afshar, M. D. Sturge, and J. S. Roberts, Phys. Rev. Lett. 77, 4792 (1996).

${ }^{2}$ C. Ell, J. Prineas, T. R. Nelson, S. Park, H. M. Gibbs, G. Khitrova, S. W. Koch, and R. Houdré, Phys. Rev. Lett. 80, 4795 (1998).

${ }^{3}$ D. M. Whittaker, Phys. Rev. Lett. 80, 4791 (1998).

${ }^{4}$ V. Savona, C. Piermarocchi, A. Quattropani, F. Tassone, and P. Schwendimann, Phys. Rev. Lett. 78, 4470 (1997).

${ }^{5}$ C. Ciuti, V. Savona, C. Piermarocchi, A. Quattropani, and P. Schwendimann, Phys. Rev. B 58, R10 123 (1998).

${ }^{6}$ J. J. Baumberg, A. Armitage, M. S. Skolnick, and J. S. Roberts, Phys. Rev. Lett. 81, 661 (1998).

${ }^{7}$ T. Baars, M. Bayer, A. Forchel, F. Schäfer, and J. P. Reithmaier, Phys. Rev. B 61, R2409 (2000).

${ }^{8}$ V. Savona and C. Piermarocchi, Phys. Status Solidi A 164, 45 (1997).

${ }^{9}$ X. Marie, P. Renucci, S. Dubourg, T. Amand, P. LeJeune, J. Barrau, J. Bloch, and R. Planel, Phys. Rev. B 59, R2494 (1999).

${ }^{10}$ G. Cassabois, A. L. Triques, F. Bogani, C. Delalande, P. Roussignol, and C. Piermarocchi, Phys. Rev. B 61, 1696 (2000).
${ }^{11}$ J. R. Jensen, P. Borri, W. Langbein, and J. M. Hvam, Appl. Phys. Lett. (to be published).

${ }^{12}$ W. Langbein and J. M. Hvam, Phys. Rev. B 61, 1692 (2000).

${ }^{13}$ E. L. Ivchenko, M. A. Kaliteevski, A. V. Kavokin, and A. I. Nesvizhskii, J. Opt. Soc. Am. B 13, 1061 (1996).

${ }^{14}$ P. Borri, W. Langbein, J. M. Hvam, and F. Martelli, Phys. Rev. B 60, 4505 (1999).

${ }^{15}$ R. P. Stanley, R. Houdré, U. Oesterle, and M. Ilegems, Solid State Commun. 106, 485 (1998).

${ }^{16}$ Due to the additional free carrier scattering, an absolute comparison between the line broadening in the microcavity and in the bare QW is difficult, and would require an accurate estimation of the free carrier density.

${ }^{17}$ V. Savona, L. C. Andreani, P. Schwendimann, and A. Quattropani, Solid State Commun. 93, 733 (1995).

${ }^{18}$ One could speculate that the absorption from the QW continuum results in an increased cavity linewidth at positive detunings, which should be included when calculating the ratio in the top part of Fig. 4. We estimated this correction and no significant changes in the trend shown in Fig. 4 were found above $25 \mathrm{~K}$. Only at lower temperatures the ratio is significantly increased by this correction and confirms the reduction of scattering processes in the LP. 